\title{
Paisaje y sociedad de alta montaña en De Madrid a Nápoles (1861) de Pedro Antonio de Alarcón
}

\section{Landscape and High-Mountain Society in Pedro Antonio de Alarcón's De Madrid a Nápoles (1861)}

\author{
Jordi Canals Piñas \\ Università degli Studi di Trento
}

\section{RESUMEN}

El extenso pasaje en el que Pedro Antonio de Alarcón describe, en De Madrid a Nápoles (1861), el territorio alpino es un valioso texto documental ya que constituye la primera descripción pormenorizada de la cadena del Mont Blanc que se registra en la tradición española de la literatura de viajes. Advertimos el propósito de contraponer un ideal de vida arcaico a la fe en el progreso material que anima a la metrópoli nacida a remolque de la revolución industrial, lo que presagia el conservadurismo ideológico de Alarcón. La descripción pone de manifiesto viejos clichés románticos, a cuya estética permanece Alarcón en parte apegado, y que se alían de manera inesperada con una visión espiritualizada de la naturaleza. Se sopesa además la huella que la guía turística Bædeker sobre Suiza, cuya 4. ${ }^{a}$ edición (1859) el autor lleva consigo en su viaje, deja en el fragmento descriptivo alpino.

Palabras Clave: Literatura de viajes; Alpes; guía turística; Baedeker; Byron; metrópoli y montaña.

\begin{abstract}
The passage from De Madrid a Nápoles (1861) in which Pedro Antonio de Alarcón describes the alpine landscape is a relevant document, as it contains the first detailed depiction of Mont Blanc's range that we come across in the history of Spanish travel literature. In the text, Alarcón sets an archaic lifestyle ideal against material progress linked to the birth of the modern metropolis under industrial revolution. This outlook anticipates the strong conservative attitude towards social changes that will increasingly be adopted by the Spanish writer in the last years of his life. From this alpine description emerge distinctive romantic clichés associated with an aesthetic dear to Alarcón that unexpectedly blend with a religious vision of nature. Furthermore, we analize the role of Bædeker's travel guide book on Switzerland (whose $4^{\text {th }}$ edition, published in 1859 , is well known to Alarcón) and the traces it has left in this extensive passage.
\end{abstract}

Key words: Travel literature; Alps; travel guide; Baedeker; Byron; Metropolis vs. Mountain. 
Pedro Antonio de Alarcón emprendió viaje a Italia el 29 de agosto de 1860 y de allí regresó el 11 de febrero de 1861, fecha en la que se encontraba ya en Madrid. En el transcurso de aquellos meses visitó las ciudades de París y Ginebra, antes de recorrer los Alpes de la Alta Saboya y el cantón suizo de Valais. Fueron estas las etapas preliminares de un viaje que tenía por meta una Italia que, en guerra con Austria y con los Estados de la Iglesia, estaba conquistando paso a paso la unidad de la nación. El testimonio de cuanto presenció en aquel largo viaje se condensa en la obra titulada De Madrid a Nápoles, cuyas dieciséis entregas se anuncian en El Museo Universal del 17 noviembre de 1861 (Palomo, 2005: LX), escasos meses después de su regreso a España.

Antes de partir, el escritor de Guadix era ya un cronista que había alcanzado una gran popularidad ${ }^{1}$. En diciembre de 1859, Alarcón se enroló en el ejército al mando de Leopoldo O’Donnell, General en Jefe de la expedición militar. En la toma de esta decisión tal vez tuvo que ver la curiosidad que le suscitaba la imagen del periodista enviado a la primera línea del frente bélico, una figura profesional que acababa de gestarse en la prensa británica a raíz del conflicto de Crimea (1853-1856)². Alarcón participó en acciones militares de la campaña de Marruecos y desde posiciones avanzadas envió a su vez crónicas que se leyeron con avidez en la Península. Buena parte del interés con el que los lectores siguieron las empresas rifeñas del ejército español cabe de hecho atribuirlo a las estrategias propagandísticas que Alarcón demostró haber asimilado con una inusitada rapidez y que constituyen «uno de los primeros ejemplos en España en que una campaña de prensa, muy bien orquestada por supuesto, determina un estado de opinión casi unánime» (Palomo, 2005: $\mathrm{X})$. Consta incluso que el guadijeño intentó innovar en el terreno de la fotografía (cuyos avances técnicos siguió desde el primer momento), por más que fueron fallidos los resultados de aquella temprana experimentación (Acosta Montoro, 1973: 250).

Los tipógrafos Gaspar y Roig, editores asimismo de la revista El Museo Universal de la que Alarcón era colaborador asiduo, recogieron en Diario de un testigo de la guerra de África (1860) el conjunto de textos escritos en Marruecos. La obra tuvo buen éxito editorial y el escritor andaluz decidió viajar acto seguido a Italia. Su propósito era el de cubrir, en calidad de testigo que iba a recorrer los territorios recién anexionados al Reino de Cerdeña, los avances de las tropas garibaldinas que estaban alcanzando las últimas victorias que llevarían a la unificación política de buena parte de la península

\footnotetext{
${ }^{1}$ Al lector que desee profundizar en la carrera periodística de Alarcón, remitimos a las páginas fundamentales de López (2008: 61-81); q. v. Palomo (2005: XXXVII-LI).

${ }^{2}$ Sobre el papel informativo clave que revistió William Howard Russell, corresponsal de The Times (el periódico londinense que no sólo formó una opinión pública, sino que la canalizó a través de sus páginas), véase Markovits (2008).
} 
italiana. La comparación implícita de la situación por la que atraviesan Francia e Italia frente a la de España, en descripciones en las que a la melancolía religiosa se mezcla una creciente desconfianza en el progreso, encubre tal vez un propósito de defensa del pensamiento unionista, tratando de contribuir a frenar el empuje disolvente de la modernidad y dando para ello su apoyo incondicional al gobierno de O'Donnell, tal como ha destacado Ignacio Javier López (2008: 179-180).

En el relato confluyen las muchas notas e impresiones personales que Alarcón tomaba a lápiz ininterrumpidamente en su cuaderno o cartera de viaje, de lo que deriva este carácter vivaz y espontáneo, tan apegado a los sucesos inmediatos. De su lectura se desprende una filosofía de viaje que, por su modernidad, sorprende al lector de hoy: Alarcón descarta la planificación meticulosa de los itinerarios y aboga por el placer de abandonarse a un vagabundeo que regula tan solo el azar o la aceptación incondicionada del riesgo ${ }^{3}$. De ahí también que su viaje, que nació con el propósito declarado de informar sobre unos acontecimientos de actualidad, suscite a menudo la impresión de buscar la línea más larga entre dos puntos geográficos.

La meta del viaje es Italia, pero en los primeros capítulos la sensación que domina al lector es la de que el periodista andaluz intenta aplazar en lo posible la llegada a aquel país. Una vez desembarcado en el puerto de Marsella, Alarcón y su compañero en este primer tramo de viaje (cuya identidad permanecerá, por cierto, en el anonimato) prosiguen viaje en ferrocarril hasta París. Tan pronto pone pie en la capital francesa, donde permanecerá «cuarenta y tantos días» (Alarcón, 1861: 61), se desplaza a Chatou para reunirse con su amigo Charles de Yriarte, al que un año antes había conocido en el transcurso de la campaña militar de Marruecos y con quien llegó incluso a compartir casa en Tetuán (Yriarte, 1863: 225-226). Aunque la intención es la de partir juntos de inmediato para Italia, deciden detenerse antes en Ginebra, aproximarse luego al valle de Chamonix y solo entonces ir al encuentro de la Italia liberada por el ejército de los Saboya. Aun así optarán por atravesar el cantón suizo de Valais, recorrer el alto valle del Ródano, superar el puerto de montaña de Simplón y tomarse un respiro a orillas del Lago Mayor antes de proseguir viaje hasta Turín. Quedarán en la capital piamontesa por espacio de una semana, transcurrida la cual ambos amigos se separarán y tomarán direcciones distintas en un intento por desentrañar por separado las claves de la tan debatida cuestión italiana.

Uniendo los puntos sobre el mapa con una línea, las etapas iniciales del viaje europeo de Alarcón nos dan como resultado un itinerario zigzagueante. En buena medida determina ese vagabundeo el capricho de la pareja de viajeros, no exento con todo de una intencionalidad que el lector, a medida que vaya

${ }^{3}$ No profundizamos en este aspecto que se encuentra desarrollado en Canals (2012). 
sumergiéndose en las páginas de este singular texto, descubrirá de manera paulatina: la necesidad que Alarcón siente de contraponer la sociedad industrializada francesa, que se está imponiendo como modelo en una Europa cegada por el progreso material, a la sociedad teocrática que está agonizando en la península italiana y de cuyas cenizas está surgiendo un nuevo Estado a orillas del Mediterráneo. Alarcón advierte la interrelación que se establece entre ambos conflictos sociales, tal como subraya en el prólogo a su primera edición y que, pese a su importancia, se omitirá en las sucesivas ${ }^{4}$ :

Úrgeme, pues, tanto conocer la cuestión de Italia como el estado de Francia. Quizás estos dos problemas se resumen en uno solo. La revolución de Italia es el volcán que revienta; pero su verdadero foco, el depósito de materias ebullicientes, está en París. Lo uno es la manifestación de lo otro. De aquí que la erupción vaya acompañada de un terremoto europeo (1861: VIII).

\section{LA METRÓPOLI PARISINA}

Alarcón se adentra en áreas geográficas del sur de Europa que, en su opinión, determinarán el rumbo político y social que emprenda, por su parte, España a partir de la segunda mitad de siglo. La ciudad de París, que en 1855 lo había deslumbrado con los avances industriales que contempló en el transcurso de su visita a la Exposición Universal (y sobre la que escribió seis crónicas que se publicaron en las páginas del periódico El Occidente durante la primavera de aquel año) ${ }^{5}$, apenas un lustro más tarde lo desengaña y se le presenta como el modelo que España debería evitar, pues el país galo encarna, en su opinión, una civilización decadente cuya inmoralidad se exhibe públicamente y sin ningún tipo de pudor (López, 2008: 85-87 y 173-179). Es en la París de la modernidad donde se produce el choque de Alarcón con el cáncer materialista que lo aterroriza, advirtiendo que el dinero se ha convertido en el patrón exclusivo con el que se regula toda relación entre los individuos que componen los distintos grupos sociales de la Francia de Napoleón III. No nos cabe duda de que la estancia parisina, a fines de aquel verano de 1860, constituirá una experiencia decisiva en el tránsito de la francofilia a la galofobia aguda que caracterizará a Alarcón a partir de este momento (Montesinos, 1977: 59-60 y 129) y que alimentará su creciente conservadurismo ideológico.

${ }^{4}$ Al proyectar la segunda edición (Madrid: 1878), Alarcón someterá el texto a una intensa operación de reescritura, de acuerdo con lo que era ya en él un hábito compositivo: «Don Pedro Antonio jamás pudo ver una nueva prueba de algo suyo sin modificarlo, alterarlo, amplificarlo o recortarlo, y ello no sólo cuando las versiones estaban separadas por largos años, sino que pudo ocurrir al cabo de pocos meses» (Montesinos, 1977: 50).

${ }^{5}$ Las ha recuperado Cyrus DeCoster (Alarcón, 1984: 126-132). Vid. Lasheras Peña (2010: 401) y Montesinos (1977: 152-153). 
A la experiencia parisina sigue — tras un brevísimo paréntesis en Ginebrala sucesiva etapa en la Alta Saboya. No es casual este orden, hasta el punto de que la huida a los Alpes permite a los viajeros zafarse de los tentáculos de la metrópoli en la que, tal como escribe el andaluz con una pizca de ironía, ha podido examinar «todos los portentos sociales» (Alarcón, 1861: 147). Las páginas en las que se describe la marcha precipitada al Valle de Chamonix cumplen, a nuestro parecer, con una función precisa en esta obra, pues sirven de pretexto al autor para contraponer dos modelos de sociedad: el de la urbe moderna, frente al enclave invulnerable y refractario al progreso del mundo exterior, donde el dinero, y hasta el mismo hombre, no han logrado destronar todavía a Dios (Alarcón, 1861: 79-83) ${ }^{6}$.

\section{El ENCUENTRO CON LOS AlPES}

Esta incursión en la colectividad montañesa tradicional, al hilo de una elección temática que juzgamos que obedece a una apuesta ideológica, hizo posible que fuera Alarcón el primer autor de nuestras letras que describió por extenso la geografía de alta $\operatorname{cota}^{7}$. Un cambio de actitud que se produjo con un notable retraso respecto al que se dio en las culturas septentrionales, en las que al entusiasmo de los autores románticos por todo cuanto implicaba un retorno a la naturaleza ${ }^{8}$ se alió la curiosidad científica de los hijos de la Ilustración (Bernard, 1978: 9).

Es en compañía del guadijeño con quien por vez primera el lector español se adentra de veras en las asperezas de los Alpes y de la cultura montañesa. Las páginas en las que el escritor andaluz describe sus aventuras alpinas en el valle de Chamonix y en el cantón de Valais revisten por ello una gran importancia, tanto desde el punto de vista del valor literario que deriva de sus precisas descripciones (escritas, por demás, por un «hombre de ojos», en feliz

\footnotetext{
${ }^{6}$ Considérese, en concreto, este pasaje de Alarcón: «Entre tanto la filosofía se esforzaba en Alemania por explicar los misterios de la creación, por razonarlo y armonizarlo todo: ¡la vida, la muerte, la eternidad, lo conocido, lo desconocido, el alma, el universo, Dios! Y uno dijo que cada hombre era un Dios; y otro que Dios no era sino la humanidad; y otros que todo era Dios y Dios era todo; y algunos que Dios no era nadie...» (1861: 79).

${ }^{7}$ El detallado registro que compiló de Beer, que incluye asimismo a Alarcón, nos permite constatar que aquel mismo año de 1860 otros viajeros célebres acudieron a la zona del Valle de Chamonix y/o Cantón de Valais, entre los que se menciona a: Erastus C. Benedict, Aimé Civiale, Emily Bliss Gould, John Ruskin, Francis Fox Tuckett, John Tyndall, Richard Denny Urlin y Edward Whymper (Beer, 1949: 284-288).

${ }^{8}$ La sugestión que el artista romántico siente ante la naturaleza virgen justifica el deseo vehemente de reintegrarse en ella, aspirando a la reconciliación con un entorno del que siente haber sido expulsado. Es un deseo que comporta de manera intrínseca la aniquilación del individuo que se entrega a la potencia devastadora de la naturaleza (Argullol, 1991: 19 y 2122; Agazzi, 2003: 409).
} 
formulación de Montesinos, 1977: 17)9 , como del manifiesto interés sociocultural que muestra. Desarrolla así motivos temáticos que se hallan presentes en la tradición alemana e inglesa, pero con escasa frecuencia en la penin$\operatorname{sular}^{10}$. De hecho no recordamos, en la literatura viajera española precedente, una crónica en la que el autor haya mostrado parecida curiosidad por la geografía y modos de vida tradicionales de las poblaciones de alta montaña como la que advertimos en el segundo capítulo de esta obra (Alarcón, 1861: 85-142). Al igual que tampoco nos consta un texto español anterior en el que el viajero haya mostrado tanta delectación ante el panorama de las estribaciones alpinas como la que percibimos en las palabras del escritor de Guadix, familiarizado desde su nacimiento con una estampa paisajística a la que las cumbres de Sierra Nevada confieren un pintoresquismo que Alarcón evocará reiteradamente a lo largo de su producción literaria.

\subsection{La montaña en la tradición hispana}

La descripción de la alta montaña es un motivo poco frecuente en la tradición literaria o pictórica española (Martínez de Pisón, 2007; Martínez de Pisón y Álvaro, 2010). Solo hacia fines del siglo XVIII se advierte una actitud nueva, fruto de una sensibilidad curtida ya en el contacto asiduo con la naturaleza, al menos por lo que se refiere a obras pertenecientes a géneros no narrativos. El canónigo canario José Viera y Clavijo (1731-1813), que a fines del Siglo de las Luces encarna una actitud científica que es paradigma de las inquietudes intelectuales de su época, manifiesta en las páginas del Diario de mi viaje desde Madrid a Italia y Alemania (publicado en 1849, aunque es el resultado de un viaje llevado a cabo a lo largo de los años 1780-1781) ${ }^{11}$ una actitud admirativa:

\footnotetext{
${ }^{9}$ Es muy atinada la distinción que en Historia de mis libros hará entre los relatos de imaginación, que «deben ser fruto de la realidad humana, sazonada por la reflexión, la filosofía y el arte», y las notas de viajero que, por su parte, «deben parecer fotografías escritas» (Alarcón, 1943: 15b).

${ }^{10}$ La travesía de los Alpes constituyó uno de los tramos culminantes del Grand Tour y también de los que suscitaban mayor emoción, máxime en un período en el que la red de comunicaciones terrestres había empezado apenas a desarrollarse. Remitimos al trabajo de Negrete Plano (2002).

${ }^{11}$ El paso por el puerto de Mont Cenis, que es una ruta que incluso en la buena estación exigía sacrificios y comportaba penalidades para el viajero, se produce exactamente el 2 de mayo de 1780. El texto de Viera y Clavijo recoge con todo detalle las operaciones que los guías llevan a cabo en Lanslebourg antes de ascender por el empinado sendero que lleva a lo alto del Mont Cenis: el despiece de los carruajes (que se volverán a montar en la primera parada que se produzca en territorio piamontés), la carga a lomos de mula y el desplazamiento de los viajeros con sillas portantinas con las que cargan los guías hasta Novalesa, el primer pueblo del Piamonte en el que se detiene la comitiva (1849: 23-24). Un desplazamiento por el corazón de la barrera alpina que hace que el erudito evoque lo que pudo ser para Aníbal aquel difícil tránsito.
} 
A pocos pasos [de Pont-de-Boivoisin] sigue una gran subida a los Alpes, y se abre a los viajantes el admirable espectáculo de estos monumentos del globo primitivo y de sus revoluciones físicas. A medida que se camina, parece que todo va inspirando entusiasmo y no sé qué agradable horror: todo es poético o filosófico (1849: 20-21).

La belleza del panorama alpino, que Viera descubrirá a medida que el grupo avance hacia el puerto del Mont Cenis (sin que ello le haga perder de vista las características geológicas de la comarca por la que atraviesa) ${ }^{12}$, no lo dejará indiferente. En su ánimo se decantarán los sentimientos antagónicos que dan fundamento a la cosmogonía romántica y sublime de la montaña: la exaltación de la belleza salvaje, de la asimetría y de cuanto carece de orden natural, del agradable horror que le suscita en definitiva aquella visión inédita ${ }^{13}$. En el transcurso del camino el viajero examinará las particularidades de una geografía para él insólita, dominada por una sucesión de altas cumbres ante las que quedará absorto:

Las faldas de estos montes eran otros montes; y, las de estos, otros y otros. De manera que durante dos horas estuvimos bajando por montes nevados y siempre descubriendo otros nuevos montes a nuestros pies. Este espectáculo es verdaderamente muy extraño y los sentidos deben probar una impresión muy nueva que deja el interior como atónito, creyéndose dotado de otro género de existencia imprevista (1849: 24).

Aun así esta descripción, que se solventa en el espacio de pocas páginas, no puede compararse con la del guadijeño. A Viera no se le presentaba otra alternativa de viaje que le permitiera evitar el puerto del Mont Cenis y su actitud es, por tanto, radicalmente distinta a la de Alarcón e Yriarte, los cuales van al encuentro premeditado del mísero enclave de Chamonix, por más que ello comporte un desvío respecto a la ruta a Italia por la vía más directa y a la que los llama el deber informativo ${ }^{14}$. La pareja de viajeros se adentra,

${ }^{12}$ Anota al salir de Modane en dirección de Lanslebourg: «en las peñas, apariencias de mármoles o jaspes» (1849: 22).

${ }^{13}$ Tales binomios antagónicos con los que describir el vértigo placentero al que sucumben quienes se aventuran por la montaña, nos hace recordar el pasaje célebre de la carta del esteta John Dennis que en verano de 1688, tras haber atravesado la barrera alpina, así escribía a su interlocutor epistolar: «We walk'd upon the very brink, in a literal sense, of Destruction; one Stumble, and both Life and Carcass had been at once destroy'd. The sense of all this produc'd different motions in me, viz. [videlicet], a delightful Horrour, a terrible Joy, and at the same time, that I was infinitely pleas'd, I trembled» (Ashfield y De Bolla, 1996: 59). La atracción por el paisaje sublime, que el irlandés Edmund Burke codificará, da razón de que quienes hasta aquel momento habían viajado para ver con sus propios ojos los restos de las antiguas civilizaciones, ascendieran ahora al Vesubio, a los valles alpinos y propiciaran de ese modo que los habitantes de enclaves recónditos como Chamonix se beneficiaran del paso de los viajeros británicos (MacFarlane, 2003: 77).

${ }^{14}$ En una de sus últimas notas redactadas en París, al calor de las noticias de la revolución garibaldina que lo sacuden del torpor en que ha caído, escribe: «La Italia antigua se desmoronaba. Muchas cosas que yo deseaba conocer iban a desaparecer para siempre. Era, pues, preciso marchar sin pérdida de tiempo» (Alarcón, 1861: 85). 
por añadidura, en la Alta Saboya en un período del año en que ya la han abandonado los turistas que todos los veranos caen sobre el valle, al hilo de la incipiente moda alpina que ha cundido sobre todo entre los burgueses de la sociedad británica ${ }^{15}$. Viajan a aquel enclave cuando, por si fuera poco, se prevén condiciones climáticas poco favorables. Nada, de hecho, los arredra; tales dificultades obran, más bien, como acicate a sus deseos compartidos de aventura.

La montaña espolea la imaginación de Alarcón, el cual permanece insensible a los numerosos atractivos seductores con los que Ginebra tienta a los viajeros. Desde el primer día de su estancia en la ciudad suiza, queda absorto en la contemplación de la cumbre del Mont-Blanc, cuya mole lejana se le impone con fuerza magnética, hasta el punto de que pierde de repente todo interés por las excursiones que los cicerones le proponen por parajes de interés turístico que se hallan a orillas del lago Lemán: «preferí las expediciones en mulo que me aguardaban en medio de las nieves: solo, libre, entregado a mis contemplaciones y luchando a cada momento con accidentes imprevistos» (1861: 94). Un deseo que se acrecienta tan pronto como se entera de que los turistas de temporada han abandonado ya el valle de Chamonix y que allí se encontrará, por tanto, en contacto íntimo con la naturaleza: «Ahora habrá allí algo inesperado, algo nuevo, algo para mí solo» (1861: 94; q. v. Canals, 2012: 45-46). Se diría que hay un arrebato espiritual al tomar la decisión que le llevará a internarse en la comarca alpina, como en busca de un arrimo primordial que intuye que solo podrá hallar al sumergirse en el aislamiento de aquel enclave de montaña ${ }^{16}$. Hay además esa querencia acentuada por los espacios naturales en los que el andaluz logra fundirse con el paisaje y obtener, a raíz de esta experiencia incluso espiritual, las que son tal vez sus descripciones más

${ }^{15}$ El texto de Alarcón constituye, también en este sentido, un testimonio excepcional. El autor ha dejado constancia de la pobreza en la que vivían inmersas las comunidades de montaña y, en concreto, de la Alta Saboya. Al pasar por Contamine-sur-Arve se conmueve al constatar la pobreza generalizada de las gentes que, al paso del carruaje, piden limosna (1861: 100). Véase también la impresión que recibe al avistar Chamonix por vez primera: «es una mísera aldea, compuesta de pobrísimas casas, en medio de las cuales se levantan cinco o seis palacios, que contrastan vivamente con el resto de la población» (1861: 105). Acto seguido dará noticias pormenorizadas sobre la explotación turística del Mont Blanc y la creciente dependencia de los habitantes respecto al flujo de viajeros procedentes del exterior.

${ }^{16}$ Se hace inevitable el recuerdo de las Lettres des deux amants (1761) de Jean-Jacques Rousseau (q. v. Ring, 2000: 16-17). Pese a que el autor no llegó a traspasar nunca la barrera montuosa, dicha obra estimuló a que generaciones de viajeros (y entre ellos también Byron y Shelley; véase Bevin, 2010: 34) decidieran aventurarse más allá de cuanto no lo hubiera hecho el propio Rousseau, contribuyendo a la fortuna turística de Suiza y propiciando, por si fuera poco, el género de la literatura de viajes: «[Switzerland] now became the goal of veritable pilgrimages. Almost all the pilgrims felt the obligation of publishing a book about their impressions; by the end of the century it is no longer possible to keep up with this travel literature» (Bernard, 1978: 19). 
logradas, lo que asomará de nuevo en sus futuras obras de viajes. Tal como ha notado Juan Luis Alborg:

es necesario leer La Alpujarra para medir su gran capacidad paisajística. Las descripciones de la naturaleza ocupan muy dilatada parte del libro; son descripciones pletóricas de detalles, pero caldeadas por el entusiasmo del viajero, siempre proclive al arrebato lírico. Pocos escritores del pasado siglo habrán sentido la hermosura de la naturaleza como el novelista de Guadix (1996: 516).

El contacto del viajero decimonónico con la geografía de alta montaña a menudo queda condicionado por un deseo de ir a la zaga de la humanidad natural, sintagma con el que aludimos a aquellos miembros de una colectividad social que resisten en un ambiente adverso y en armonía con el entorno sin que ningún agente externo haya alterado su modo de ser originario. Al científico y poeta Albrecht von Haller se atribuye el sentar las bases del culto por los Alpes, a raíz del viaje que llevó a cabo en 1728 y cuyas observaciones propician un poema de intención didáctica: Die Alpen (Berna: 1732). En el relato de aquel viaje, dará igual peso a la observación de la naturaleza que de la población que reside en los valles suizos en la que ve un residuo puro y no contaminado por la corrupción que emanaba de la ciudad y que atribuye a la nefasta influencia de la civilización francesa (Bernard, 1978: 11-12). Una coincidencia notable, como se advierte, con la actitud que mostrará Pedro Antonio de Alarcón, quien insistentemente contrapondrá la autenticidad y pureza de los habitantes idealizados de los Alpes a la falsedad y servilismo del hombre moderno.

La pervivencia de una humanidad natural es una idea recurrente en la temprana literatura europea de montaña, lo que llevó a la consecuencia de que ya desde el siglo XVIII Suiza se erigiera en símbolo de la cultura alpina y meta de los primeros viajeros de montaña (Giacomoni, 2003: 28). No es, con todo, un concepto restringido a dicha área geográfica, sino que asoma incluso en la incipiente tradición científica española del siglo XIX con referencia a comarcas escasamente exploradas. Lo percibimos, por poner un ejemplo, en las palabras con que Casiano de Prado describe a los pastores que habitan las aldeas cercanas a la garganta del río Cares: «cuya vida en todas las estaciones es una continua lucha, pero que se arrostra con calma y como una condición ordinaria que impone allí al hombre la naturaleza en sus admirables armonías» (1855: 11). El paisaje montañés se configura, en definitiva, como enclave de difícil acceso en el que sobrevive una colectividad que encarna un estilo de vida antagónico al de quienes viven en poblaciones de valle (y, por supuesto, en la metrópoli), y que al mismo tiempo se hace portadora de unos modos de vida arcaicos que se hallan en simbiosis total con el medio ambiente.

Es esta también una de las claves interpretativas que se desprende de la lectura del relato del viaje de Alarcón. En su obra el guadijeño enfrenta de una manera neta la civilización pujante de los parisinos, que como hemos visto se 
erige en modelo amenazador para la civilización occidental, al estilo de vida de las poblaciones alpinas de la Alta Saboya. Tal visión alarconiana, que opone el progreso a la cultura de montaña, sintoniza con las ensoñaciones alpinas de George Gordon Byron que se hallan contenidas en el Childe Harold's Pilgrimage (1812-1818) que el escritor de Guadix incrusta en su texto al verter en prosa versos que extrae de diversos lugares del Canto Tercero (estr. 62, 68, 69, 72 y 85) ${ }^{17}$. En tales fragmentos Byron ensalza el alcázar alpino y sus murallas heladas como lugar que le permite olvidar el tumulto de las ciudades que se le ha hecho de repente intolerable, tras caer en la cuenta de la vanidad de su vida anterior, cuando gustaba de «los mugidos del Océano furioso» (Alarcón, 1861: 96), mientras que hoy se complace en la navegación sosegada por la superficie de las aguas silenciosas del Lago Lemán.

De por sí no resulta en exceso sorprendente que Alarcón adopte como guía la poesía romántica de lord Byron, pues a mediados del siglo XIX se ha consolidado la costumbre de que el turista se sirva del «libro poético de viajes» que se vincula a determinados lugares y que lo acompaña en su desplazamiento, convirtiéndose en filtro a través del cual observa la realidad que le rodea $(\mathrm{Bu}-$ zard, 1993: 110-111; Bevin, 2010: 155-156). Es un hecho documentado que los versos de Byron se contaban entre los predilectos de la masa de turistas británicos que viajaban a Europa, y que hicieron que el lord inglés se erigiera en arquetipo del turista atípico que el viajero, que no deseaba confundirse con la masa, podía adoptar como modelo y guía sin que ello le creara ningún tipo de conflicto de conciencia (Buzard, 1993: 118-122). Se ha considerado que el retorno a la naturaleza que anhela Byron en parte de su producción va más allá de aspectos lingüísticos y formales (la adjetivación no se desmarca de la que utilizaron autores de la tradición precedente) ${ }^{18}$; sí tendrá, en cambio, consecuencias temáticas de mayor alcance, como las que supone la revisitación del gótico arquitectónico en cuya apreciación tanto peso había jugado, también para John Ruskin, la reiterada experiencia alpina: «When describing the

${ }^{17}$ El mismo Alarcón admite ser el traductor del fragmento, como declara al poner término a la larga cita y apelar al consabido tópico de la falsa modestia: «Si obedeciese a mis inclinaciones, seguiría traduciéndoos a lord Byron hasta el fin de su poema, con lo que ganaríais mucho más que con mi prosa desbarajustada» (1861: 96). Presumiblemente traduce del francés, dado el desconocimiento de la lengua inglesa que admite en más de un lugar a lo largo de esta misma obra.

${ }^{18}$ Léase cuanto anota, al respecto, Marjorie Hope Nicolson: «The critics did not seem to notice that the pattern of such poems as The Excursion, Alastor, and Childe Harold's Pilgrimage had been established in the "excursion" poems of Mallet, Savage, and Thomson, that the details of the Romantic mise-en-scène mountains, caverns, cataracts, ruins, hurricanes, storms, volcanoes, earthquakes had been the stock in trade of eighteenth-century descriptive poets, or that the characteristic adjectives and epithets of the Romantic poets had long been familiar: dark, gloomy, still, awful, unfathomable, ghastly, rude; magnificent, majestic, grand; glorious; terror and delight, joy and exultation. If there was "something strange" in Romantic Nature description, it lay elsewhere than in pattern, scene, vocabulary» (1959: 21). 
architecture of the North, Ruskin is conscious of the analogy, and notes that there is a fraternal relationship between the wild mountains and the Gothic edifices» (Colley, 2010: 169).

El de la arquitectura que se proyecta sobre el paisaje que atraviesa el viajero es un binomio al que tampoco Alarcón se muestra insensible, habida cuenta de los pasajes en los que las montañas son obras que se asocian a motivos arquitectónicos religiosos: «Las escarpadas puntas que forman sus cúspides [de las Agujas de Varens], brillan al sol como plateados capiteles» (1861: 102); o también cuando al pasar por la carretera que lleva al puerto de Simplón escribe «Así se camina horas enteras, bajo techado y de balcón en balcón, produciéndoos la extraña impresión que os causaría veros llevado en diligencia por los claustros altos de un convento o por la galería de un palacio» (1861: 135). La vegetación alpina coadyuva asimismo a la consolidación de este motivo: «El camino flanqueaba un monte cubierto de pinos, que a veces formaban una bóveda sobre nuestra cabeza» (1861: 103).

\subsection{Naturaleza edénica y religión}

Hemos aludido al arrebato romántico que incita al andaluz a abandonar repentinamente Ginebra e ir al encuentro de una naturaleza primaria, auténtica, esencial y alejada de la colectividad metropolitana. En esta decisión impulsiva entra en juego el desengaño que le provoca el contacto con las sociedades desarrolladas y que hace que se acentúe su animadversión por todo cuanto es obra del individuo que se complace en la modernidad. De ahí que marche en búsqueda de un mundo edénico y solitario en el que todo obedezca al designio de Dios y en el que sus escasos pobladores se fundan armónicamente con el entorno natural, sin que estos desvirtúen la obra de la Creación. El viaje a los Alpes constituye en el fondo un viaje al pasado, a un lugar impermeable a los vientos de la modernidad, a una sociedad montañesa que se ajusta a los dogmas de una religiosidad tradicional (y son de hecho numerosas las analogías con cuanto encontrará en su sucesiva incursión por la Alpujarra), en la que sus componentes sufren con resignación la pobreza y permanecen inmunes a la influencia de las peligrosas reivindicaciones de los obreros de las ciudades. De algún modo es un texto que aboga por el inmovilismo social, pese a que Alarcón ha tomado conciencia de que no será posible detener el avance del progreso.

Tal ideario fue tomando forma en el transcurso de su estancia en París. Sobre todo a raíz de una escena, solo en apariencia intrascendente, de la que fue testigo al regreso de una excursión a Saint-Denis: el transporte, en un carro tirado por cuatro bueyes, de un árbol corpulento que los empleados de la municipalidad habían arrancado de un bosque distante con la intención de trasplantarlo en el Jardín de las Tullerías «para que diera sombra a un banco de pie- 
dra que estaba muy expuesto al sol» (1861: 76). Alarcón advierte en esta acción una cifra de la soberbia del hombre que atenta contra la voluntad de Dios, que suplanta al Hacedor y que crea un mundo artificial a su medida, aunque ello comporte el tener que violentar a la naturaleza. Todo lo cual le hace concluir:

Cuando en adelante penetre yo en un bosque, en busca de soledad y de misterio, ya no me infundirán veneración los amores de la naturaleza, el afán con que el árbol se agarra a la madre tierra, la piedad con que la cubre de sombra y de frescura, el apoyo y compañía que da a las flores y a las yerbas silvestres, ni el acuerdo y la reciprocidad de beneficios con que viven en sociedad aves y hojas, reptiles y musgos, perfumes y rocíos, auras y rumores... ¡No! Me diré: ¡todo esto es mentira! ¡ todo es invención humana! Vosotros, elementos de la vida, no os conocéis ni os amáis. Y acaso tú, frondoso árbol que me albergas, eres en este bosque un desterrado como yo, un extranjero solitario, un alma en pena perdida en el desierto (1861: 76).

El anhelo por una naturaleza auténtica se vincula a su querencia por la religión primitiva. Son actitudes que, en cierto modo, simbolizan el rechazo de lo espurio y el ansia de autenticidad que determinan las elecciones del viajero.

La sociedad de alta montaña de la que Alarcón es testigo en otoño de 1860 es muestra anacrónica de conservadurismo social, pues en ella todo individuo ocupa el lugar y la escala jerárquica que siempre ocupó. Dicha colectividad vive en uno de los pocos lugares del mundo occidental que no ha quedado a merced de los vaivenes que agitan a las sociedades urbanas europeas, que por aquellos mismos años han sucumbido a la fe ciega en el progreso material. De ahí también el hecho de que los Alpes se presenten a los ojos de Alarcón, así como de buena parte de los viajeros que le han precedido, como un espacio virginal, como una persistencia inviolada del universo originario creado por Dios. Son todos ellos motivos que Alarcón reitera y desarrolla en la poesía en cuartetos endecasílabos que lleva por título «El Mont-Blanc», fechada en Chamonix en 1860 aunque publicada una década más tarde en Poesías serias y humorísticas (Alarcón, 1870: 73-77), compuesta por tanto a lo largo de aquel mismo viaje que llevó al guadijeño a un contacto incipiente con el reino de la alta montaña. En ella la cumbre más alta de la cadena alpina, junto a la que el resto de montes que la rodean son apenas «escabeles de su trono inmenso» (v. 30) y en la que todo yace empequeñecido a sus pies, se le presenta al escritor errante y cosmopolita como lugar que forma parte de la «mansión eterna» y que por tanto le aleja de las tribulaciones de la Tierra ${ }^{19}$.

\footnotetext{
${ }^{19}$ Se ha considerado que el viaje o peregrinación que describe en La Alpujarra (1873), en plena Semana Santa, constituye una ascensión mística que persigue el encuentro con Dios, fundiéndose con la belleza de las asperezas de Sierra Nevada (Lucena, 2006: 157-159). Pese a los doce años que transcurrieron entre la publicación de De Madrid a Nápoles y La Alpuja$r r a$, hay una neta continuidad ideológica.
} 
Trasluce dicho poema una actitud romántica que lleva a valorar la montaña, el océano o el desierto como lugares en los que el poeta aspira a poder refugiarse en su huida de la sociedad y donde poder cancelar las huellas de su paso por un mundo en agitación: «desparecer, huyendo de la tierra / desde esta cima que se acerca al cielo; / por siempre desertar de aquella guerra, / de eterna libertad tendiendo el vuelo» (vv. 61-64). Hay un byronismo que ha sedimentado en la posición de Alarcón ante la existencia, aunque contrarrestado todo ello por la actitud religiosa e incluso mística (pues allí experimenta la ataraxia del ánimo reacio al «raudo movimiento» [v. 37] del mundo) que a partir de estos años caracterizará progresivamente la ideología de Alarcón. Es el Mont Blanc el paraje agreste donde el poeta experimentará un «horror profundo» (v. 49) y primordial, inherente a toda fusión con la naturaleza alpina de acuerdo con una visión de la montaña que remonta sobre todo a Joseph Addison, quien se cuenta entre los primeros tratadistas que emparejaron la sublimidad (o greatness, de acuerdo con su formulación; véase Addison, 1854: 327-328; q. v. Ashfield y De Bolla 1996: 58-71) al paisaje, en especial el de montaña, lo que permeará sucesivamente el ideario estético de Edmund Burke y de Kant; pero es también donde sentirá, por fin, la exaltación de hallarse en una mayor proximidad con Dios: «ufano aquí mi corazón palpita... / ¡Aquí! ¡Solo con Dios...! ¡Fuera del mundo! / ¡Solo, bajo la bóveda infinita!» vv. 5052). Esta actitud religiosa, tan acusada y que en ocasiones tanto recuerda a la del joven John Ruskin (Bevin, 2010: 64-66), es la que lleva al ensimismamiento del guadijeño con el entorno alpino y en la que radica tal vez su mayor discrepancia con aquella actitud romántica con la que antaño se había identificado.

\section{LA GUÍA TURÍSTICA BAEDEKER}

Alarcón viaja a las regiones alpinas con la misma intencionalidad nostálgica y romántica con la que años más tarde llevará a cabo sus incursiones por las provincias remotas de la Península Ibérica «donde pervivían usos milenarios que una época prosaica y utilitaria no había podido desterrar» (Montesinos, 1977: 241). Las consecuencias de esta decisión tienen para el lector contemporáneo un valor que trasciende este propósito, pues como se ha dicho el relato del accitano contiene la que es la primera descripción literaria pormenorizada de un entorno de montaña en lengua española. Alarcón se postula como intérprete de esta realidad geográfica con la que nuestras letras se hallan tan escasamente familiarizadas y de esa opción derivan consecuencias de naturaleza lingüística que pueden calibrarse desde perspectivas heterogéneas: si, por un lado, quedan incrustados en el texto los calcos o préstamos tomados del francés, habida cuenta de la fuente bibliográfica de la que parte, Alarcón se esforzará en otros casos por proponer equivalentes léxicos castizos a 
los realia que impone la descripción de un entorno natural extremo al que él se aproxima por experiencia directa, pero mediando también la lectura de la obra que le sirve de guía en su travesía alpina.

Así, por ejemplo, no duda en adoptar el préstamo fr. glacier $^{20}$ para designar un fenómeno natural que, como veremos, maravillará al escritor andaluz hasta el punto de describirlo por extenso en su obra. Se servirá, explicitando en este caso su fuente bibliográfica directa (regla que no siempre respetará), de la guía que Karl Bædeker escribió sobre Suiza y que constituye uno de los títulos de mayor fortuna de la etapa editorial ligada al incipiente fenómeno del turismo de masas: La Suisse, les lacs italienes, Milan, Turin, Gênes et Nice. Manuel du voyageur (1852) $)^{21}$. Para el término fr. névé renuncia asimismo a dar con un equivalente castizo (es más, niega que pueda corresponderse con el esp. nevero, pese a la semejanza léxica) pues, tal como precisa el guadijeño, no hay en España cumbres que superen la altura necesaria. Da equivalentes para algunas voces con uso específico en la geología (esp. mesa y esp. embudo), sin dar noticia en estos casos del sustantivo francés del que parte para su traducción. Estrategia que sí empleará, en cambio, para fr. crevasse $\rightarrow$ esp. grieta, por más que recurrirá de manera reiterada al préstamo a lo largo del fragmento sin insistir en la correspondiente equivalencia (p. ej. en: «nada es más fácil que poner el pie sobre una crevasse y ser engullido por ella», Alarcón, 1861: 114). Con actitudes muy distintas se enfrenta, como vemos, a la dificultad léxica que entraña la descripción de un entorno que para él resultaba insólito.

${ }^{20}$ La voz glaciar no se registra en textos españoles anteriores al de Alarcón y tampoco, como vemos, en este autor, pese a que CREA proporciona documentación sacada de $D e$ Madrid a Nápoles que en realidad, juzgando por las ediciones de 1861 y 1878 , no tiene lugar. Dicho sea de paso, el texto de Alarcón es también el documento español más antiguo, de los que componen el corpus de CREA, en el que se registra la voz fr. glacier que nunca antes se había tomado en préstamo para incrustarla en un texto en lengua castellana.

${ }^{21}$ De dicha traducción francesa hubo en el siglo XIX un total de 21 ediciones. Postulamos que Alarcón lleva en su viaje la cuarta, a la que recurrimos en este trabajo (Baedeker, 1859). Para reconstruir las vicisitudes editoriales de las guías Bædeker, resulta de utilidad el siguiente enlace http://www.bdkr.com/index.php [consultado el 31/10/2013]. Mucho antes el editor británico John Murray se había aproximado al pintoresquismo de Suiza en su A Glance at Some of the Beauties and Sublimities of Switzerland (1829), interés que se consolidó con el inicio de la publicación de las primeras guías propiamente dichas a partir de 1836 (Sillitoe, 2004), y que descartamos que Alarcón pudiera consultar. Sobre este título inicial de Murray ha escrito Bevin (2010: 34-35): «began a series of handbooks on Switzerland and the Alps described as the «first popular guidebook to the region», a view endorsed by [Albert] Smith himself». La Alta Saboya y Suiza se convertirán en meta del turismo masivo a fines de junio de 1863, fecha en la que un grupo de sesenta viajeros emprende el primer viaje organizado obviamente por Thomas Cook al Valle de Chamonix y a los cantones helvéticos (Brendon, 1991: 79-83). Ha llegado hasta nosotros el diario de Jemima Morrell (1963), una de las participantes en la primera expedición alpina, que ha servido a Diccon Bewes (2013) como punto de partida para una reflexión sobre los efectos del turismo de masas en el desarrollo económico y cultural de la Confederación Helvética. 
Hemos anticipado la dependencia de Alarcón a publicaciones de carácter turístico. El trasvase de pasajes de la guía Bædeker a la crónica de Alarcón pone en entredicho la afirmación del autor en el prólogo de 1861 respecto a que el texto es producto de cuanto él ha visto sin ningún tipo de intermediación:

No concluiré, sin embargo, antes de deciros, por lo que pueda valer, que yo no pienso contaros sino aquello que haya visto por mis propios ojos y tocado con mis propias manos. Y que si en mis mejores excursiones he cometido la atrocidad de dejar de ver una cosa muy importante, la cosa muy importante se quedará por decir. $\mathrm{Y}$ que si he tenido la desgracia de no encontrar en alguna parte lo que esperaba, no me figuraré que lo he encontrado, ni lo contaré de oídas o leídas (1861: X).

Pese a que se encarniza, acto seguido, en la tendencia de Alejandro Dumas al plagio en sus relatos de viaje, cuyos hábitos de producción editorial censura una y otra vez, no procede Alarcón de manera distinta respecto a la guía que tan de auxilio le resulta para sus descripciones, sobre todo para las de aquellos lugares por los que pasa de manera apresurada sin detenerse, a bordo de un medio de transporte que le impide el contacto directo con las poblaciones que atraviesa. El hecho de que en su descripción quede en la mayor parte de casos constancia del territorio intermedio entre el lugar de partida y la meta de llegada favorece que el guadijeño opte por este auxilio. Una vez llegado a Chamonix, en cambio, tomará la palabra y describirá con voz propia cuanto observe a su alrededor. La fuente Bædeker es, de alguna manera, el cañamazo sobre el que se entrecruzan numerosos pasajes de la descripción alpina en la obra de Alarcón. El texto fuente proporciona los datos esenciales que el guadijeño toma tal cual o que en algunos casos contados amplifica. Se configura, pues, como el texto nuclear a partir del cual puede expandir su descripción.

Otras veces el recuerdo de la fuente textual le da pretexto para introducir un motivo que juzga adecuado a la descripción. Es el caso, por ejemplo, de cuando ascienden a Montanvert desde donde contemplarán el Mar de Hielo: la lectura de que en Caillet muchos árboles han sido arrastrados por los aludes (noticia que se trasvasa tal cual al texto de Alarcón: "A michemin, Caillet, source d'eau fraîche, autrefois ombragée d'arbres qui ont été entraînés par la chute des neiges», 1859: $185 \rightarrow$ «Ya hemos pasado por Caillet, en otro tiempo cubierto de árboles que los aludes han arrastrado en su caída», 1861: 110) le incita a hablar por extenso de estas temibles avalanchas que constituyen un riesgo para los excursionistas.

Si por lo general hay un trasvase directo, operación que trasluce sobre todo a nivel léxico, cabe señalar también la existencia de pasajes que entrañan una visión subjetiva de la realidad circunstante que no sólo se mantiene, sino que se acentúa en el texto de Alarcón. Así, por ejemplo, el entusiasmo que comparte con Bædeker al cruzar la frontera con Italia tras dejar a las espaldas la garganta de Gondo, y la exaltación al poner por fin los pies en la italiana Val d'Ossola: 
La vue en est saissante; c'est une contrée décidément italienne, quoiqu'elle ait beaucoup souffert des inondations dans les dernières années. Tout différe de la vallée du Rhône, air balsamique, teintes de l'horizon, verdure, vignes en berceau, gros épis de maïs, villages avec leurs maisons blanches éparses sur la plaine; tout annonce l'Italie, jusq'au cri-cric du grillon et au coassement des grenouilles (1859: 214) $\rightarrow$ ¡Oh, sí...! ¡Aquella es Italia! Aquel cielo turquí, aquel fulgurante sol, aquella riente campiña cruzada por plateados ríos, aquellas verdes colinas coronadas de blancos palacios, aquellos olivares oscuros, aquellas praderas de esmeralda, aquellas graciosas quintas, todo aquello es lo que yo me imaginaba desde niño... ¡Cuánto fulgor en el espacio! ¡Qué matices en la llanura! ¡Qué perfumes en el aire! ¡Qué temperatura tan amorosa después del frío que hemos pasado! Allí reina aún la primavera... Las viñas ostentan todavía sus pámpanos, los árboles sus verdes hojas, el sol su creadora llama, el ambiente sus gérmenes de vida. ¡Cómo se comprende que esta tierra sea tan codiciada! ¡Cuán bella la verían todos los conquistadores al asomar por los Alpes! ¡Cuán hermosa la encontrarán sus hijos cuando vuelvan a hallarla después de un largo destierro! (1861: 140).

En la traducción del largo fragmento en el que Bædeker se aproxima al fenómeno glacial, Alarcón se ve constreñido a soluciones de compromiso. No evita cuanto comporte búsqueda de construcciones perifrásticas y de analogías al alcance de la comprensión del lector español que sugieran con mayor naturalidad un fenómeno parecido: «Cette neige grenue» (1859: XLI) $\rightarrow$ «Esta nieve, que cae en forma de granizo» ${ }^{22}$ (1861: 111). Detectamos también un intento de trasvase del texto original a un registro que Alarcón imaginaba más fácilmente comprensible por parte del lector medio. Considérese, en el sintagma siguiente, la puesta en relación de la dureza con la condensación del hielo: «une glace moins condensée» (1859: XLII) $\rightarrow$ «el hielo menos duro» (1861: 111). A este intento de dar con una formulación llana para un fenómeno complejo creemos que obedece también la siguiente solución: «Aussitôt que la neige du névé a atteint la limite du point de congélation» (1859: XLII) $\rightarrow$ «Ahora bien: cuando la nieve del névé [...] llega a la región del hielo» (1861: 111).

Pese a que se supone que la de Alarcón es una versión castellana muy respetuosa del texto fuente (Alarcón llega incluso a marcar con las comillas el inicio y el final del pasaje traducido, así como con las fórmulas inicial y conclusiva de cita: «He aquí su explicación [de K. Bædeker]», 1861: 111, y «Hasta aquí Bædeker», 1861: 114), el cotejo pone al descubierto la omisión de diversos pasajes. Aquellos, por ejemplo, en los que con una visión enciclopédica el autor de la guía introduce en su discurso consideraciones lingüísticas y una tabla de equivalencias para el fenómeno glaciar ${ }^{23}$ :

${ }^{22}$ Aunque cabe también la duda de que sencillamente está adoptando una estrategia de traducción que le permite obviar la dificultad al desconocer la terminología específica: fr. neige grenue $\rightarrow$ esp. nieve granular.

${ }^{23}$ La visión de los glaciares le impactará de modo indeleble, hasta el punto de que en el futuro su imagen servirá como paragón: los ríos de lava que ve al viajar en ferrocarril hacia Pompeya «Me recuerdan los glaciers de Suiza» (1861: 632). El fenómeno glaciar fue de 
on l'appelle en all. Gletscher, ital. ghiacciaio («giäsch»), d'où vient peut-être la dénomination de Kess dans la Carinthie et le Salzburg; dans le Tyrol, Ferner; sur le versant italien Vedretto, dans les Grisons Wader, dans le Valais biegno, dans le Piémont et la Savoie ruise (1859: XLI).

En la versión española desaparecen, de manera generalizada, las huellas léxicas del texto alemán original que habían quedado en la traducción francesa de la guía, mientras que en contrapartida añade Alarcón consideraciones léxicas que juzga relevantes para lectores españoles:

Cette tête neigeuse et non condensée du glacier se nomme névé (en all. Firn) (1859: $X L I I) \rightarrow$ Esta nieve suelta, o no condensada, que se encuentra a la cabeza del glacier se llama névé (palabra que no tiene equivalente en español, a causa de que en España no hay alturas que excedan de 10.000 pies) [...] (al cual [névé] no hay que confundir tampoco con nuestros ventisqueros [...]) (1861: 112).

Esta atenuación de la carga informativa de tipo enciclopédico que llevaba consigo la guía turística que le sirve de texto fuente, se hace perceptible también en aquellos casos en los que en Bædeker se describen procesos geológicos complejos, como son la fusión del hielo que alimenta la corriente de agua que fluye por debajo de la masa glaciar:

Dans les régions inférieures, la fonte du glacier produit aussi de l'eau; cette eau s'évapore de même ou s'infiltre dans les profondes crevasses et se confond avec l'eau de source qui coule dans le lit du glacier et forme le ruisseau qui s'en écoule (1859: XLII) $\rightarrow$ el derretimiento continuo de sus capas inferiores produce un arroyo y hasta un río, que corre siempre por debajo de todo glacier (1861: 112-113).

Advertimos, en el citado fragmento transcrito, que el andaluz simplifica todo el proceso de evaporación o de filtración por las profundas grietas, según se describía en la guía, tras lo cual el agua derretida se suma a la que fluye por el lecho del glaciar y forma un río, mientras que Alarcón apunta sintéticamente a la fusión del hielo y a la formación de la corriente subterránea.

El escritor accitano da una idea sintética de la explicación exhaustiva que, en el texto fuente, se proporciona sobre los factores que condicionan la velocidad a la que fluye el glaciar hacia el fondo del valle destacando la lentitud con la que la masa de hielo desciende, evitando de ese modo desconcertar al lector con la imagen de la velocidad irregular que el autor de la guía registra, en lo que tal vez podamos interpretar como una discrepancia respecto a la fuente: «[les glaciers] s'avancent ici plus vite, là plus lentement» (1859: $\mathrm{XLII}) \rightarrow$ «[los glaciers $]$ avanzan lentamente sobre los valles» (1861: 113). Alarcón ignorará de hecho los párrafos que en Bædeker siguen a este enunciado, ricos en documentación científica relativa al desplazamiento de la masa

hecho, en la práctica totalidad de viajeros que quedaron subyugados por la belleza del territorio de los Alpes, el motivo paisajístico de mayor incitación al conocimiento de la alta montaña (MacFarlane, 2003: 103-136; Negrete Plano, 2002: 107 y 110). 
de hielo, mencionando para ello las conclusiones que son fruto de la observación de las investigaciones de Forbes, Saussure y Agassis, que se describen en el texto fuente con gran detalle hasta el punto de incluir largas citas literales de sus respectivos tratados científicos.

Otro rasgo perceptible en la versión castellana es la eliminación de redundancias, como si Alarcón fuera a la zaga de una economía del relato, omitiendo aquellos datos que le parecen secundarios en el texto que traduce. Le resulta superfluo, al parecer, mencionar por segunda vez que se refiere al hielo del glaciar, pues resulta obvio al lector que sigue tratando del mismo:

[Les glaciers primaires] Les glaciers primaires sont de longues masses de glace, comparables à un fleuve glacé; ils s'étendent le long des vallées, n'ont souvent qu'une pente insensible et se composent d'une glace ferme, dense et qui atteint jusqu'à 1500 d'épaisseur (1859: XLI-XLII) $\rightarrow$ [Los glaciers primarios] son largas masas de hielo, semejantes a un río helado, que se extienden a lo largo de los valles, con una ligera inclinación, y que llegan a medir algunas veces 1.000 y 1.500 pies de espesor o profundidad! (1861: 111).

Esta solución comporta la pérdida de dos notas adjetivas («glace ferme, dense»), que tal vez juzga Alarcón que no sean en exceso significativas. Un caso análogo advertimos en el pasaje siguiente, donde el complemento circunstancial se precisa por dos veces con variante, mientras que en el texto meta la pareja bimembre de sintagmas queda reducida a un único elemento: «[Les glaciers secondaires] ils sont comme suspendus aux parois des vallées, aux flancs des montagnes» (1859: XLI-XLII) $\rightarrow$ «están como colgados de los flancos de las montañas» (1861: 111).

No duda el escritor andaluz en enmendar lo que juzga que son incoherencias del texto fuente, sometiendo el texto a una reescritura que le permite dotar a la descripción de mayor lógica. Considérese el fragmento siguiente:

Des plus petits objets, comme des pierres, une feuille ou même un insecte mort agissent sur la glace d'une manière inverse. Le soleil les réchauffe; la glace qui est au-dessous d'eux se fond, et il se forme ainsi des entonnoirs (1859: XLIII) $\rightarrow$ provienen de haber caído sobre aquel un ave muerta, un objeto de metal, o una piedra de cierta forma o tal naturaleza, cuyos objetos, calentados por el sol, derriten la nieve que tienen debajo (1861: 113).

Aquí se han reemplazado algunos de los objetos que, al caer sobre el hielo, favorecen la creación de los famosos embudos. Ya no son, como se leía en el pasaje fuente, 'piedras, hojas o insectos muertos', sino 'piedras, objetos de metal o aves muertas'. Tal vez se le hace difícil justificar que un simple insecto haya podido provocar esta apertura en el hielo, mientras que la sustitución por un pájaro hace más creíble la hipótesis científica. Asimismo por lo que se refiere al reemplazo de las hojas caídas sobre la superficie del glaciar con los objetos de metal que puedan dejar a sus espaldas los ocasionales excursionistas. Se esfuerza además, en este pasaje, por dar coherencia al proce- 
so de formación del embudo, por lo que amplifica el entero fragmento para poder de ese modo ilustrarlo con mayor eficacia al lector profano.

Pese a que Bædeker es la fuente primaria, hay pasajes del texto alarconiano que no guardan relación con la fuente y que contienen información que nos hace postular que el guadijeño pudo haber consultado otras obras y fundir los datos con el largo fragmento que constituye una traducción literal (aunque con ocasionales divergencias) de la guía a la que recurre. La sospecha es la de que el conocimiento personal, fruto de su observación directa y de las explicaciones de los guías que acompañan a los viajeros hasta el mirador, ha propiciado la reelaboración del texto fuente. Compárese la amplificación en el fragmento siguiente:

Cette curiosité s'explique par ce fait que la glace environnante a été fondue par le soleil, tandis que la dalle a servi de parasol au pilier qui la soutient (1859: XLIII)

Este curioso fenómeno se explica perfectamente. De los flancos de las montañas cae sobre el glacier una piedra extensa y delgada: el sol derrite con el tiempo la nieve alrededor de aquella losa, pero no la nieve que hay debajo de ella, y a la cual sirve como de sombrilla: y al cabo de algunos meses, la piedra queda en el aire, tendida sobre un pilar de hielo, que adelgaza continuamente hasta que se rompe, y que entre tanto marca el alto nivel a que llegó la nevada en tal o cual invierno (1861: 113).

La imagen del texto original no le parece tal vez lo suficientemente ajustada a la realidad (o, por lo menos, a la realidad que él ha contemplado con sus propios ojos), por lo que introduce observaciones personales que enriquecen la descripción: «c'est ce qui forme les pyramides et les aiguilles de glace» (1859: XLIV) $\rightarrow$ «De este modo se producen las graciosas agujas de hielo, las pirámides y todas las demás caprichosas figuras que sorprenden al observador» (1861: 113).

Hemos considerado ya (por lo que se refiere a las variantes que se ve forzado a introducir el traductor en busca de coherencia) un pasaje que Alarcón modifica de manera radical, reemplazando elementos que permiten dar una explicación más plausible a fenómenos glaciares. No satisfecho con ello, y preocupándose de que el lector pueda entender el proceso de formación de los embudos, amplifica de modo notable el entero pasaje:

Des plus petits objets, comme des pierres, une feuille ou même un insecte mort agissent sur la glace d'une manière inverse. Le soleil les réchauffe; la glace qui est au-dessous d'eux se fond, et il se forme ainsi des entonnoirs (1859: XLIII) $\rightarrow$ Otra rareza de la superficie de los glaciers, es lo que se llama un embudo, el cual es un agujero de la forma que indica su nombre. Estos agujeros, que a veces horadan todo el glacier y llegan a comunicarse con el río que corre por su lecho, provienen de haber caído sobre aquel un ave muerta, un objeto de metal, o una piedra de cierta forma o tal naturaleza, cuyos objetos, calentados por el sol, derriten la nieve que tienen debajo. Es la razón contraria a la que explica la existencia de las mesas. Siempre que hay derretimientos, el agua se filtra por estos embu- 
dos, y naturalmente, va fundiendo a su paso un hielo secular a que no hubiera llegado nunca la acción del sol (1861: 113).

Intuimos que Alarcón va en pos de un estilo discursivo. Frente a pasajes en los que hay yuxtaposición de acciones, el accitano opta por una sintaxis más compleja que entraña subordinación o coordinación. Adapta de ese modo la lengua que se está configurando como característica de la naciente guía turística $^{24}$ a la complejidad lingüística del texto literario. Compárense, al respecto, los siguientes lugares:

les nuages en se déchargeant déposent sur la terre de la neige et non de la pluie. Cette neige grenue s'amoncelle dans les gorges; l'aire et le soleil en amollissent la surface, surtout dans les parties les plus basses; la nuit, cette neige condensée gèle, et est recouverte par une nouvelle neige (1859: XLI) $\rightarrow$ nieva siempre en vez de llover. Esta nieve, que cae en forma de granizo, se amontona en los barrancos, donde el aire y el sol ablandan algo su superficie, y forman de ella una capa compacta, que se hiela a la noche, y se ve pronto cubierta por otra nueva capa de nieve (1861: 111).

Les glaciers primaires sont de longues masses de glace, comparables à un fleuve glacé; ils s'étendent le long des vallées (1859: XLI-XLII) $\rightarrow$ Los glaciers primarios son largas masas de hielo, semejantes a un río helado, que se extienden a lo largo de los valles (1861: 111).

Des plus petits objets, comme des pierres, une feuille ou même un insecte mort agissent sur la glace d'une manière inverse. Le soleil les réchauffe; la glace qui est au-dessous d'eux se fond, et il se forme ainsi des entonnoirs (1859: XLIII) $\rightarrow$ provienen de haber caído sobre aquel un ave muerta, un objeto de metal, o una piedra de cierta forma o tal naturaleza, cuyos objetos, calentados por el sol, derriten la nieve que tienen debajo (1861: 113).

Introduce marcadores del discurso de carácter distributivo con los que amalgamar el discurso, fundirlo en un todo entrelazado y continuado, frente a la sintaxis enumerativa del texto original caracterizada por oraciones yuxtapuestas: «Par l'action de l'air et du soleil sur la surface du glacier, celui-ci s'évapore. Dans les régions inférieures, la fonte du glacier produit aussi de l'eau» (1859: $\mathrm{XLII}) \rightarrow$ «En primer lugar, el sol y el aire, obrando sobre la superficie del glacier, producen una gran evaporación; y, por otro lado, el derretimiento continuo de sus capas inferiores produce un arroyo» (1861: 112-113). Mientras que con otros marcadores realza realidades de carácter opuesto: «Quand le courant du

\footnotetext{
${ }^{24}$ Son escasos los lugares en los que Alarcón procede en dirección contraria. Recordamos aquí un fragmento en el que se introduce en la versión castellana del texto, y sin correspondencia con la fuente, características prescriptivas propias del género editorial de la guía: «Mais quand une neige fraîchement tombée recouvre la crevasse, un seul faux pas peut entraîner une chute mortelle dans l'abîme» (1859: XLIV) $\rightarrow$ «También suele acontecer, en las grandes nevadas, que las grietas se llenen de nieve, en cuyo caso nadie debe aventurarse a reconocer el glacier, pues nada es más fácil que poner el pie sobre una crevasse y ser engullido por ella...» (1861: 113-114).
} 
glacier est forcé de franchir le bord d'un escarpement, sa masse solide se déchire en fragments innombrables sur lesquels agissent l'air et le soleil» (1859: XLIII-XLIV) $\rightarrow$ «Por la inversa: cuando la corriente lenta del glacier se ve obligada a pasar sobre un terreno escarpado, la masa cristalina se rompe en mil fragmentos, sobre los que obran después el sol y el aire» (1861: 113).

El trasvase a un registro literario del texto en el que aparecen numerosas marcas linguiísticas propias de la guía, se evidencia también en el refuerzo de la expresividad que se registra en el texto meta. Cuanto era descripción neutra, queda recubierto por una descripción modalizada que se refleja tipográficamente en el recurso a signos de exclamación ausentes en el texto fuente: «[Les glaciers primaires] n'ont souvent qu'une pente insensible et se composent d'une glace ferme, dense et qui atteint jusqu'à 1500 d'épaisseur» (1859: XLI-XLII) $\rightarrow$ «[Los glaciers primarios] con una ligera inclinación, y que llegan a medir algunas veces 1.000 y 1.500 pies de espesor o profundidad!» (1861: 111). En otro caso, Alarcón introduce marcas lingüísticas que refuerzan la aseveración de cuanto se ha descrito: «il faut nécessairement que l'augmentation soit compensée par une diminution quelconque» (1859: XLII) $\rightarrow$ «hácese necesario que el aumento se compense con alguna disminución. Y así es» (1861: 112).

\section{CONCLUSIONES}

En Historia de mis libros Pedro Antonio de Alarcón se hará eco, a inicios de noviembre de 1884 , de la fortuna editorial que había alcanzado la recopilación de crónicas periodísticas o «de caminante», tal como las denominará al término de su carrera en el tan discutido balance del conjunto de su producción (1943: 15b). Deja allí constancia de que la obra a la que nos hemos acercado es el resultado de los apuntes, escritos «con lápiz, muy extensamente y d'après nature» (1943: 15b) en el transcurso de su deambular por Francia y Suiza antes de dirigirse a una Italia levantada aún en armas y en lucha por la unificación política del territorio. Son notas cuyo autor escribe a menudo de manera espontánea en su cartera de viaje; y esto, al margen del valor testimonial que confiere al texto, nos permite seguir la evolución de un ideario moldeado por los acontecimientos del intelectual que es testimonio de un período histórico convulso.

En el curso de su viaje por Europa, Alarcón se siente espoleado a reflexionar sobre un nuevo modelo de sociedad que avanza imparable a remolque de la revolución industrial. Pasa, en cambio, a un segundo plano, el deseo de ir a la zaga de emociones estéticas o con el propósito formativo de contemplar en Italia las obras clave de la latinidad o admirar los logros artísticos del Renacimiento, objetivo predominante en otros muchos relatos coetáneos de viajeros. Su visión es la del observador que a mediados del s. XIX se desplaza por un continente europeo que se le presenta como un laboratorio político 
y social. La prolongada estancia en París, el sucesivo tránsito por la Europa alpina, el viaje a los territorios unificados de Italia y la entrada en la Roma pontificia de Pío IX constituyen al respecto incursiones en colectividades humanas que encarnan fenómenos sociales heterogéneos e incluso excluyentes, algo que el propio Alarcón explicita en el prólogo a la primera edición del texto.

A sus lectores ofrece un retrato desengañado y pesimista del panorama político europeo, ante el que la sociedad española vive de espaldas, indiferente a la amenaza que se cierne sobre ella. Significativamente el largo relato, en el que se condensa una experiencia de viaje que se ha prolongado por más de cinco meses, concluye con el retorno del autor a un Madrid inmerso en la despreocupación de las fiestas de Carnaval. Las del mismo año, por cierto, que funcionará como marco espacial de El escándalo $(1875)^{25}$, donde contrapone el desasosiego existencial de Fabián Conde a la muchedumbre que invade las calles madrileñas en aquel 11 de febrero de 1861.

Las páginas en las que Alarcón se aproxima con curiosidad a los enclaves alpinos saboyano y suizo ocupan el espacio intermedio comprendido entre la descripción de las mencionadas colectividades antagónicas: el patrón francés, que se impone a grandes pasos en el continente europeo, y la anacrónica teocracia romana. Una vez en los Alpes el guadijeño se deja sugestionar por aquel entorno agreste, sin limitarse a esbozar meros apuntes paisajísticos. Su mirada va más allá y se centra sobre todo en la pervivencia de unos modos de vida tradicionales de los que se siente testigo último, advirtiendo que peligran aquellos enclaves de alta montaña en los que, si bien no se ha roto todavía el vínculo espiritual que une al individuo con la naturaleza, sus pobladores están sucumbiendo ya progresivamente al mercantilismo ${ }^{26}$ :

No bien llega el verano, Chamounix se llena de ingleses ${ }^{27}$ y todos los habitantes del valle se convierten en guías, y todos los mulos encuentran quien los alquile para subir a los montes, y las vacas dan leche y queso, y sus tiernas hijas se truecan en chuletas o beefsteak, y todas las manufacturas del invierno encuentran salida, y el oro inglés cae como el maná sobre la comarca (1861: 105).

${ }^{25}$ Tal como anota Ignacio Javier López en su edición crítica del texto alarconiano: «en El escándalo el coro desempeña un papel antagónico. Aparece representado en el desorden, o «infierno» del Carnaval; y el protagonista, a contracorriente de la multitud, aparece desde el comienzo enfrentado a su ambiente y al mundo exterior» (Alarcón, 2013: 179, n. 2).

${ }^{26}$ Advertimos una irritación parecida a la que sentía John Ruskin, para quien la montaña tomó un carácter sacral, hasta el punto de que no toleraba que se transformara en vulgar mercancía (Quinsac, 2003: 175). La creciente afluencia de los turistas le llevó a tomar conciencia de ser uno de los últimos testigos de un paisaje que estaba condenado a transformarse y al que, en los años finales de su vida, volvería tan sólo en el recuerdo (Bevin, 2010: 76-78 y 124-125).

${ }^{27}$ De nacionalidad británica fueron de hecho los pioneros del alpinismo y también quienes empezaron a ocupar, en los meses de verano, los escasos establecimientos hoteleros del valle de Chamonix (Ring, 2000). 
Ciertamente es la de Alarcón una actitud contrapuesta a la de la mayor parte de sus coetáneos que, fiados del avance del progreso, no advierten los riesgos que acarrea el espejismo de la revolución industrial.

A la toma de conciencia de Pedro Antonio de Alarcón, a quien urge dejar constancia escrita de una sociedad que claudica, deben las letras españolas una de las descripciones más logradas del entorno alpino.

\section{BIBLIOGRAFÍA CITADA}

Acosta Montoro, José (1973). «Inquietos y viajeros: Pedro A. de Alarcón y Ernest Hemingway», en Periodismo y literatura, II. Madrid: Ediciones Guadarrama, pp. 239-272.

Addison, Joseph (1854). «Essays on the Pleasures of the Imagination», en The Works of Addison, VI. The Spectator. New York: G.P. Putnam \& Co., pp. 322-373.

Agazzi, Elena (2003). «Vette ambigue. Immagini letterarie della montagna tra Settecento e Novecento», en Gabriella Belli, Paola Giacomoni y Anna Ottani Cavina (ed.), Montagna. Arte, scienza, mito da Dürer a Warhol. Milano: Skira Editore, pp. 409-420.

Alarcón, Pedro Antonio de (1861). De Madrid a Nápoles. Madrid: Impr. y Libr. de Gaspar y Roig.

Alarcón, Pedro Antonio de (1870). Poesías serias y humorísticas. Madrid: Tipografía de Gregorio Estrada.

Alarcón, Pedro Antonio de (1943). «Historia de mis libros», en Obras completas. Luis Martínez Kleiser (ed). Madrid: Ediciones Fax, pp. 3-28.

Alarcón, Pedro Antonio de (1984). Obras olvidadas. Cyrus DeCoster (ed.). Madrid: José Porrúa Turanzas.

Alarcón, Pedro Antonio de (2013). El escándalo. Ignacio Javier López (ed.). Madrid: Ediciones Cátedra.

Alborg, Juan Luis (1996). «Pedro Antonio de Alarcón», en Historia de la literatura española, 5.1. Realismo y naturalismo: La novela. Madrid: Gredos, pp. 480-588.

Argullol, Rafael (1991). La atracción del abismo. Un itinerario por el paisaje romántico. Barcelona: Ediciones Destino.

Ashfield, Andrew y Peter De Bolla (ed.) (1996). The Sublime. A Reader in British EighteenthCentury Aesthetic Theory. Cambridge: Cambridge University Press.

Baedeker, Karl (1859). La Suisse, les lacs italienes, Milan, Turin, Gênes et Nice. Manuel du voyageur. Coblenz: K. Bædeker Editeur.

Beer, Gavin Rylands de (1949). Travellers in Switzerland. London: Oxford University Press.

Bernard, Paul Peter (1978). Rush to the Alps. The evolution of vacationing in Switzerland. New York: Columbia University Press.

Bevin, Darren (2010). Cultural climbs. John Ruskin, Albert Smith and the alpine aesthetic. Saarbrücken: VDM Verlag Dr. Müller.

Bewes, Dicon (2013). Slow Train to Switzerland. London-Boston: Nicholas Brealey Publishing.

Brendon, Piers (1991). Thomas Cook. 150 Years of Popular Tourism. London: Secker \& Warburg.

Buzard, James (1993). The Beaten Track. European Tourism, Literature, and the Ways to Culture, 1800-1918. Oxford: Clarendon Press.

Canals, Jordi (2012). «La dicotomía turista / viajero en De Madrid a Nápoles (1861) de Pedro Antonio de Alarcón», Pasos. Revista de Turismo y Patrimonio Cultural. 10, 4, pp. 39-48.

Colley, Ann C. (2010). Victorians in the Mountains: Sinking the Sublime. Surrey: Ashgate. 
Giacomoni, Paola (2003). «"Dare del tu alle rocce”», en Gabriella Belli, Paola Giacomoni y Anna Ottani Cavina (ed.), Montagna. Arte, scienza, mito da Dürer a Warhol. Milano: Skira Editore, pp. 19-39.

Hope Nicolson, Marjorie (1959). Mountain Gloom and Mountain Glory. Ithaca: Cornell University.

Lasheras Peña, Ana Belén (2010). España en París. La imagen nacional en las Exposiciones Universales (1855-1900). Santander: Universidad de Cantabria.

López, Ignacio Javier (2008). Pedro Antonio de Alarcón (prensa, política, novela de tesis). Madrid: Ediciones de la Torre.

Lucena, Joanne (2006). «Alarcón’s Pilgrimage: La Alpujarra», Crítica Hispánica. 28, 2, pp. $147-160$.

MacFarlane, Robert (2003). Mountains of the Mind. A History of a Fascination. London: Granta Books.

Markovits, Stefanie (2008). «Rushing into Print: "Participatory Journalism" during the Crimean War», Victoria Studies. 50, 4, pp. 559-586.

Martínez de Pisón, Eduardo (2007). «Viajeros a las montañas», en José M. Oliver, Clara Curell, Cristina G. Uriarte y Berta Pico (ed.), Escrituras y reescrituras del viaje. Miradas plurales a través del tiempo y de las culturas. Bern: Peter Lang, pp. 333-346.

Martínez de Pisón, Eduardo y Sebastián Álvaro (2010). El sentimiento de la montaña. Doscientos años de soledad. Madrid: Desnivel.

Montesinos, José F. (1977). Pedro Antonio de Alarcón. Madrid: Castalia.

Morrell, Jemima (1963). Miss Jemima's Swiss Journal. The First Conducted Tour of Switzerland. London: Putnam.

Murray, John (1829). A Glance at Some of the Beauties and Sublimities of Switzerland. With Excursive Remarks on the Various Objects of Interest, Presented during a Tour through its Picturesque Scenery. London: Longman-Rees-Orme-Brown and Green.

Negrete Plano, Almudena (2002). «Suiza y el paso de los Alpes en los recuerdos del Westmorland», en José María Luzón Nogué (ed.), El Westmorland. Recuerdos del Grand Tour. Sevilla: Fundación Cajasol, pp. 107-117.

Palomo, María del Pilar (2005). «Introducción», en Pedro Antonio de Alarcón, Diario de un testigo de la guerra de África. Sevilla: Fundación José Manuel Lara, pp. IX-LXXXV.

Prado, Casiano de (1855). Valdeón, Caín, La Canal de Trea. Ascensión a los Picos de Europa, en la Cordillera Cantábrica. [S.1.]: [s.n.].

Quinsac, Annie-Paule (2003). «Scienza, etica, «speculum dei»: la montagna nell'opera di John Ruskin», en Gabriella Belli, Paola Giacomoni y Anna Ottani Cavina (ed.), Montagna. Arte, scienza, mito da Dürer a Warhol. Milano: Skira Editore, pp. 171-176.

Ring, Jim (2000). How the English Made the Alps. London: John Murray.

Rousseau, Jean-Jacques (1761). Lettres de deux amans, habitans d'une petite ville au pied des Alpes. Amsterdam: Marc Michel Rey.

Sillitoe, Alan (2004). Leading the Blind. A Century of Guide Book Travel (1815-1914). Nottingham: Bookcase Editions.

Viera y Clavijo, José (1849). Estracto de los apuntes del diario de mi viaje desde Madrid a Italia y Alemania. Santa Cruz de Tenerife: Imprenta, Litografía y Librería Isleña.

Yriarte, Charles (1863). Sous la tente. Souvenirs du Maroc. Récits de guerre et de voyage. Paris: Morizot.

Fecha de recepción: 26 de noviembre de 2013

Fecha de aceptación: 28 de mayo de 2014. 